\title{
Phenotypic Characterization of Local Chicken Ecotypes of Benishangul-Gumuz Region, Western Ethiopia
}

\author{
Habtamu Alebachew ${ }^{1 *} \quad$ Alemayehu Abebe ${ }^{1} \quad$ Fekadu Begna $^{1} \quad$ Kedja Ahmed $^{1}$ \\ Alayu Tarekegn $^{1} \quad$ Dessie Abera $^{1} \quad$ Diribi Mijana $^{2}$ \\ 1.Ethiopian Institute of Agricultural Research, Assosa Agricultural Research Center, P.O.Box 265 \\ 2. Ethiopian Institute of Agricultural Research, Wondogenet Agricultural Research Center, P.O.Box 198
}

\begin{abstract}
This study was conducted in four districts (Bambassi, Kamashi, Mao-komo and Homosha) of Benishangulgumuz regional state to describe physical and morphological chrematistics of local chicken population in the study area. A total of 847 matured local chickens (619 females and 228 males) were randomly sampled from the study area. Mean live body weight and other linear body measurements of males was significantly $(\mathrm{p}<0.05)$ higher than females. The overall mean values of measured traits males of indigenous chicken for body length, wingspan, and height and body weight were $37.0 \pm 4.26 \mathrm{~cm}, 22.9 \pm 2.72$, and $41.9 \pm 5.47 \mathrm{~cm}$ and $1.5 \pm 0.413 \mathrm{~kg}$ respectively. While the respective values for mature females were $34.0 \pm 3.19 \mathrm{~cm}, 21.4 \pm 2.72 \mathrm{~cm}, 36.6 \pm 2.98 \mathrm{~cm}$ and $1.3 \pm 0.32 \mathrm{~kg}$. Almost all chickens (91\%) in the study area were feathered neck. Regarding the head shape, $81 \%$ of the chicken in the study area had plain head shape and the remaining $(19 \%)$ were crest. Single comb was the most common (82\%) comb type and was predominant in all of the districts. The study revealed that most of the parameters measured revealed distinctive variations, providing the basis for further characterization of local chicken breeds; therefore future study can be concentrated on selection for qualitative traits of interest.
\end{abstract}

Keywords: Benishangulgumuz: Local chicken; Phenotypic characterization; Qualitative variation; Quantitative variation;

DOI: $10.7176 / \mathrm{JBAH} / 9-7-03$

Publication date: April $30^{\text {th }} 2019$

\section{Introduction}

Ethiopia possesses huge number of chicken population in Eastern Africa. According to CSA (2015) chicken population in the country estimated to be 56.87 million of which 96 percent are indigenous chicken ecotypes. Chicken population in Benishangulgumuz is estimated to be 1.38 million; from this 0.62 million, 0.56 million, 0.12 million and 0.06 million are found in Assosa, Metekel, Kamash zones and Maokomo special district, respectively.

Even though chicken population is huge in Ethiopia, the production system majorly is still very traditional that chickens are not provided with enough feed, poor health and housing managements. However, despite this improper management, indigenous chickens are resistance to harsh environments, tolerant to diseases and provide better test of meat and eggs than exotic ones (Taddele and Ogle, 2001). Daikwo et al. (2011) stated that indigenous chickens in rural areas of tropics manifest great deal of variations due to genetic and environmental factors, thus they are reservoirs of genetic materials for genetic studies, improvement, preservation and conservation. The same is true for Ethiopia as the country is endowed with varied agro-ecological zones and owns diverse animal genetic resources. There has been a trade with Arab and Asian countries since long time ago, and thus these waves of trade and movements of people and animals have influenced the genetic make-up of domestic animals including chickens (Workneh, 1992). Then, generally the naming of these indigenous animals was based on either the area occupied by the animals or ethnic groups or clans keeping them (Halima, 2007).

Improvement of local chicken productivity through selection and cross breeding is vital for all

developing countries especially for Ethiopia since there is dynamic increment of human

population, and incompatibility of demand and supply of animal protein (Solkner et al., 2008). To improve the performance of indigenous chicken identification of available genetic resource is important (FAO, 2011 and Rege et al., 2011). Different studies have been done so far to characterize indigenous chickens of Ethiopia (Tadelle, 2003; Halima, 2007; Nigussie et al., 2010; Addisu et al., 2014, Embet et al., 2014, Agide, 2015 and Feyera, 2016). These studies have not covered Benshangul-gumuz Regional State. In the absence of this information, it is difficult to design chicken breeding strategies and program for the region. Therefore, the objective of this study was to identify, characterize and describe the phenotypic variations of indigenous chicken populations in Benshangul-gumuz regional State of Ethiopia by taking qualitative and quantitative morphological traits.

\section{Materials and Methods}

Description of the study areas $\square$ The study was conducted in four districts (Bambassi, Kamashi, Homosha and Maokomo) of Benishangulgumuz regional state. Assosa town is located at $670 \mathrm{~km}$ west of Addis Ababa, capital 
city of Ethiopia. Bambasi is located $45 \mathrm{~km}$ East of Assosa town, whereas Kamashi, Homosha and Maokomo are located $225 \mathrm{~km}$ North East, $35 \mathrm{~km}$ West and $105 \mathrm{~km}$ South West of Assosa town, respectively.

Benishangulgumuz regional state is located between geographical coordinates of $9^{\circ} 30^{\prime} \mathrm{N}$ to $11^{\circ} 39^{\prime} \mathrm{N}$ latitude and $34^{\circ} 20^{\prime} \mathrm{E}$ to $360^{\circ} 30^{\prime} \mathrm{E}$ longitude with altitude ranging from 1272 - 1573 masl (AsARC, 2006). Mean annual rainfall and temperature of the region lies between $700-1450 \mathrm{~mm}$ and $21-35^{\circ} \mathrm{C}$, respectively (AMS, 2008).

Phenotypic measurements and observations: Linear body measurements and other physical characteristics were measured and observed from 847 chickens, comprising of 228 males and 619 females. The measurement was taken from matured local chicken greater than 6 months of age by asking chicken owners. Measurements were taken early in the morning to avoid the effect of feeding and watering on the chicken size and conformation. Qualitative traits such as plumage colour, body shape, comb type, shank colour, skin colour, head shape, ear lobe colour and eye colour was documented through direct visualization. Whereas measurable traits such as body weight $(\mathrm{kg})$, body length, wing span, shank length, breast circumference, wattle length and width, keel length, beak length, comp length and width, toe to back length, tail length, earlobes length and width and height were measured using spring balance and centimetre $(\mathrm{cm})$ in the nearest two 0.5 digits using breed characterization manual ( FAO,2012).

Statistical analysis: SAS, 2008 -program version 9.2 and SPSS (Version 20) were used for all statistical analysis in this study

\section{Qualitative Morphological Traits}

Univariate Analysis: Qualitative morphologic traits were subjected to frequency procedure of SPSS (Version20).

\section{Quantitative morphological traits}

Univariate Analysis: Quantitative morphological traits were subjected to analysis of variance using the general linear model procedure (PROC GLM) of SAS, 2008 version 9.2 to detect statistical differences among sample chicken populations. Duncan's New Multiple Range Test mean comparisons were made for variables showing significant differences between sample populations. Taking districts ecotypes and sex of the chicken as main fixed effects, the following model was used.

$Y i j=\mu+D i+S j+A S i j+\varepsilon i j k$ Where;

$Y i j=$ The observed linear measurements

$\mu=$ Overall mean

$D i=$ Fixed effect of $\mathrm{i}^{\text {th }}$ district/ecotype (i=1 Bambassi, 2=Kamashi, 3= Mao-komo, $4=$ Homosha)

$S j=$ Fixed effect of $\mathrm{j}^{\text {th }}$ sex $(\mathrm{j}=$ male or female $)$

$A D i j=$ Fixed effect of interaction of $\mathrm{i}^{\text {th }}$ district/ecotype with $\mathrm{j}^{\text {th }}$ sex

$\varepsilon i j k=$ random error

\section{Results and discussion}

\section{Variation in quantitative traits}

Least square means \pm SD of body weight $(\mathrm{kg})$ and other linear body measurements $(\mathrm{cm})$ for all districts and sex are shown in Table 1. All the quantitative dependent variables were significantly $(\mathrm{p}<0.05)$ affected by sex of chickens. The live body weight and other body measurements of this study also showed that male chicken population was significantly higher $(\mathrm{P}<0.05)$ than female chicken population in the study areas. This shows the presence of sexual dimorphism in the population. The sexual dimorphism is explained by the differences in level of male sex hormones which is responsible for greater muscle development in males than in females (Jansson et al., 1985).

The mean measured values of body length, back length, wing span, breast circumference and keel length for local adult hens in the study areas were $35.3,1.5,22.7,35.8$ and $9.6 \mathrm{~cm}$ respectively, with body weight of $1.3 \mathrm{~kg}$. The overall mean body weight of adult hens in this study is similar with the study of Eskindir et al. (2013) who reported $1.29 \mathrm{~kg}$ for indigenous chicken population of Horro district in East Wellega zone of Oromia region, Ethiopia. But, it is higher than $1.10 \mathrm{~kg}$ reported by Addis (2014) for indigenous hens in eastern Amahara region of Ethiopia. However, the study by Addisu et al. (2013) for indigenous chicken population of the same sex in north Gondor zone of Ethiopia indicated higher body weight $(1.37 \mathrm{~kg})$ and wing span $(36.25 \mathrm{~cm})$. Variation in body weight and other liner body measurements in the present study compared to the literature could be attributed to the ecotype differences among various indigenous chicken populations of Ethiopia.

The mean body weight and body length of local adult cocks in the study area were $1.5 \mathrm{~kg}$ and $37.8 \mathrm{~cm}$, respectively which are close to body weight of $1.5 \mathrm{~kg}$ and body length of $38.0 \mathrm{~cm}$ of indigenous chicken population of Horro and Jarso districts of Ethiopia reported by Eskindir et al. , (2013). The study by Addis et al. 
(2014) for indigenous cocks in eastern Amahara region of Ethiopia, however, indicated lower value of body weight $(1.10 \mathrm{~kg})$ and body length $(35.8 \mathrm{~cm})$. But, Halima (2007) reported higher value of body weight $(2.02 \mathrm{~kg})$ for adult cocks in Northwest Ethiopia.

In this study, cocks and hens in Kamashi district were found to be significantly higher $(\mathrm{P}<0.05)$ in body length, back length, keel length and height compared to the other chicken populations in the study districts. These higher values could be associated with environmental hotness of Kamashi district compared to others as these morphological traits are important for better heat dissipation in the tropical hot environment (Nesheim et al., 1979).

Table 1.Morphometric variations of local chickens in the study districts (in $\mathrm{cm}$ )

\begin{tabular}{|c|c|c|c|c|c|c|c|c|c|c|}
\hline \multirow{2}{*}{\multicolumn{2}{|c|}{$\begin{array}{l}\text { Parameter } \\
\text { Variables }\end{array}$}} & \multicolumn{4}{|c|}{ Districts/ecotypes } & \multirow{2}{*}{$\begin{array}{l}\text { Over all } \\
(\text { mean } \pm \text { SE }) \\
\mathrm{F}=619, \mathrm{M}=2 \\
28\end{array}$} & \multirow{2}{*}{$\begin{array}{l}\text { Grand } \\
\text { mean } \\
\text { mean } \pm \mathrm{SE}) \\
\mathrm{N}=847\end{array}$} & \multirow{2}{*}{$\mathrm{P}_{\mathrm{D}}$} & \multirow{2}{*}{$\mathrm{P}_{\mathrm{S}}$} & \multirow{2}{*}{$\mathrm{P}_{\mathrm{DxS}}$} \\
\hline & & Bambassi & Kamashi & Mao-komo & Homosha & & & & & \\
\hline \multirow{2}{*}{$\begin{array}{l}\text { Comb } \\
\text { length }\end{array}$} & $M$ & $48+195 a$ & $51+203 a$ & $43+193 a$ & $45+239$ & $47+202$ & \multirow{2}{*}{$2.9 \pm 1.2$} & \multirow{2}{*}{ * } & \multirow{2}{*}{$* * *$} & \multirow{2}{*}{ * } \\
\hline & $\mathrm{F}$ & $2.2 \pm 0.67 \mathrm{a}$ & $2.2 \pm 0.69 \mathrm{a}$ & $2.1 \pm 0.74 a$ & $2.1 \pm 0.59 \mathrm{a}$ & $2.2 \pm 0.68$ & & & & \\
\hline \multirow{2}{*}{$\begin{array}{l}\text { Comb } \\
\text { width }\end{array}$} & $\mathrm{M}$ & $1.8 \pm 1.1 \mathrm{~b}$ & $2.5 \pm 1.39 \mathrm{a}$ & $1.9 \pm 1.1 \mathrm{~b}$ & $1.9 \pm 1.3 \mathrm{~b}$ & $2.0 \pm 1.26$ & \multirow[t]{2}{*}{$1.1 \pm 0.77$} & \multirow[t]{2}{*}{$* *$} & \multirow[t]{2}{*}{$* * *$} & \multirow[t]{2}{*}{$* * *$} \\
\hline & $\mathrm{F}$ & $0.61 \pm 0.52 \mathrm{a}$ & $0.71 \mathrm{a}$ & $0.71 \pm 0.48 \mathrm{a}$ & $0.70 \mathrm{a}$ & $0.70 \pm 0.48$ & & & & \\
\hline \multirow{2}{*}{$\begin{array}{l}\text { Wattle } \\
\text { length }\end{array}$} & $\mathrm{M}$ & $2.7 \pm 0.87 \mathrm{ab}$ & $2.9 \pm 1.08 \mathrm{a}$ & $2.4 \pm 0.95 \mathrm{~b}$ & $2.4 \pm 1.0 \mathrm{~b}$ & $2.6 \pm 0.97$ & \multirow[t]{2}{*}{$1.7 \pm 0.61$} & \multirow[t]{2}{*}{$* *$} & \multirow[t]{2}{*}{$* * *$} & \multirow[t]{2}{*}{$* * *$} \\
\hline & $\mathrm{F}$ & $1.5 \pm 0.45 \mathrm{a}$ & $1.5 \pm 0.35 \mathrm{a}$ & $1.4 \pm 0.41 \mathrm{a}$ & $1.4 \pm 0.35 \mathrm{a}$ & $1.4 \pm 0.39$ & & & & \\
\hline \multirow{2}{*}{$\begin{array}{l}\text { Wattle } \\
\text { width }\end{array}$} & $\mathrm{M}$ & $2.0 \pm 0.95 \mathrm{ab}$ & $2.2 \pm 1.23 \mathrm{a}$ & $1.7 \pm 1.05 \mathrm{~b}$ & $1.8 \pm 1.23 \mathrm{~b}$ & $1.9 \pm 1.11$ & $0.97 \pm 0.67$ & $* *$ & $* *$ & ** \\
\hline & $\mathrm{F}$ & $0.6 \pm 0.47 \mathrm{a}$ & $0.6 \pm 0.40 \mathrm{a}$ & $0.7 \pm 0.40 \mathrm{a}$ & $0.6 \pm 0.31 \mathrm{a}$ & $0.6 \pm 0.41$ & & & & \\
\hline Beak & $\mathrm{M}$ & $1.6 \pm 0.17 \mathrm{a}$ & $1.5 \pm 0.37 \mathrm{ab}$ & $1.5 \pm 0.21 \mathrm{ab}$ & $1.6 \pm 0.15 \mathrm{a}$ & $1.6 \pm 0.25$ & $1.5 \pm 0.22$ & $* * *$ & $* * *$ & ns \\
\hline length & $\mathrm{F}$ & $1.5 \pm 0.37 \mathrm{a}$ & $1.4 \pm 0.24 \mathrm{~b}$ & $1.3 \pm 0.21 \mathrm{c}$ & $1.5 \pm 0.16 \mathrm{a}$ & $1.5 \pm 0.21$ & & & & \\
\hline Beak & $\mathrm{M}$ & $0.66 \pm 0.12 \mathrm{a}$ & $0.7 \pm 0.20 \mathrm{a}$ & $0.64 \pm 0.15 a$ & $0.7 \pm 0.18 \mathrm{a}$ & $0.7 \pm 0.16$ & $0.6 \pm 0.15$ & $*$ & $* * *$ & ns \\
\hline width & $\mathrm{F}$ & $0.62 \pm 0.10 \mathrm{ab}$ & $0.6 \pm 0.14 \mathrm{~b}$ & $0.62 \pm 0.18 \mathrm{ab}$ & $0.65 \pm 0.13 \mathrm{a}$ & $0.6 \pm 0.14$ & & & & \\
\hline Wing & $\mathrm{M}$ & $20.5 \pm 1.75 \mathrm{a}$ & $19.7 \pm 1.84 b$ & $20.5 \pm 1.69 a$ & $19.8 \pm 2.41 \mathrm{ab}$ & $20.1 \pm 1.92$ & $19.3 \pm 1.7$ & $* * *$ & $* * *$ & ns \\
\hline length & $\mathrm{F}$ & $19.3 \pm 2.1 \mathrm{a}$ & $18.6 \pm 1.58 \mathrm{~b}$ & $19.31 \pm 1.53 \mathrm{a}$ & $18.7 \pm 1.46 \mathrm{~b}$ & $18.9 \pm 1.68$ & & & & \\
\hline Wing spin & $\mathrm{M}$ & $24.2 \pm 3.50 \mathrm{ab}$ & $25.2 \pm 2.86 \mathrm{a}$ & $24.3 \pm 4.10 \mathrm{a}$ & $22.9 \pm 2.72 b$ & $24.2 \pm 3.34$ & $23.1 \pm 3.03$ & $* * *$ & $* * *$ & ns \\
\hline & $\mathrm{F}$ & $22.8 \pm 2.95 \mathrm{a}$ & $23.4 \pm 2.83 a$ & $22.9 \pm 3.54 \mathrm{a}$ & $21.4 \pm 2.18 \mathrm{~b}$ & $22.7 \pm 2.91$ & & & & \\
\hline Body & $\mathrm{M}$ & $36.5 \pm 4.02 b$ & $39.8 \pm 4.79 a$ & $37.6 \pm 4.16 b$ & $37.0 \pm 4.26 \mathrm{~b}$ & $37.8 \pm 4.32$ & $35.9 \pm 3.59$ & $* * *$ & $* * *$ & ns \\
\hline length & $\mathrm{F}$ & $34.3 \pm 2.49 \mathrm{c}$ & $36.5 \pm 3.70 \mathrm{a}$ & $35.5 \pm 3.37 \mathrm{~b}$ & $34.0 \pm 3.19 \mathrm{c}$ & $35.31 \pm 3.29$ & & & & \\
\hline Back & $\mathrm{M}$ & $17.4 \pm 2.25 \mathrm{~b}$ & $21.8 \pm 3.49 \mathrm{a}$ & $17.1 \pm 3.10 \mathrm{~b}$ & $17.2 \pm 2.18 \mathrm{~b}$ & $18.5 \pm 2.82$ & $17.6 \pm 2.67$ & $* * *$ & $* * *$ & ns \\
\hline length & $\mathrm{F}$ & $16.2 \pm 2.38 \mathrm{~b}$ & $19.8 \pm 3.01 \mathrm{a}$ & $15.6 \pm 2.43 b$ & $16.1 \pm 2.36 \mathrm{~b}$ & $17.3 \pm 2.26$ & & & & \\
\hline EL & $\mathrm{M}$ & $2.3 \pm 0.76 \mathrm{ab}$ & $2.6 \pm 2.31 \mathrm{a}$ & $1.9 \pm 0.68 \mathrm{~b}$ & $1.9 \pm 0.56 \mathrm{~b}$ & $2.2 \pm 1.43$ & $1.7 \pm 0.97$ & $* * *$ & $* * *$ & $*$ \\
\hline & $\mathrm{F}$ & $1.5 \pm 0.39 \mathrm{a}$ & $1.6 \pm 1.15 \mathrm{a}$ & $1.5 \pm 0.65 \mathrm{a}$ & $1.5 \pm 0.39 \mathrm{a}$ & $1.5 \pm 0.73$ & & & & \\
\hline EW & $\mathrm{M}$ & $1.34 \pm 0.60 \mathrm{a}$ & $1.3 \pm 0.61 \mathrm{ab}$ & $1.2 \pm 0.58 \mathrm{ab}$ & $1.1 \pm 0.55 \mathrm{~b}$ & $1.2 \pm 0.59$ & $0.9 \pm 0.40$ & $*$ & $* * *$ & $* *$ \\
\hline & $\mathrm{F}$ & $0.8 \pm 0.26 \mathrm{ab}$ & $0.7 \pm 0.29 \mathrm{~b}$ & $0.8 \pm 0.32 \mathrm{a}$ & $0.8 \pm 0.33 \mathrm{ab}$ & $0.8 \pm 0.30$ & & & & \\
\hline SL & $\mathrm{M}$ & $8.1 \pm 0.89 \mathrm{a}$ & $7.8 \pm 1.11 \mathrm{a}$ & $7.8 \pm 1.11 \mathrm{a}$ & $7.8 \pm 1.20 \mathrm{a}$ & $7.9 \pm 1.07$ & $6.9 \pm 0.89$ & $*$ & $* * *$ & ns \\
\hline & $\mathrm{F}$ & $6.8 \pm 0.94 a$ & $6.5 \pm 0.70 \mathrm{~b}$ & $6.6 \pm 0.85 \mathrm{ab}$ & $6.6 \pm 0.81 \mathrm{ab}$ & 6.70 .82 & & & & \\
\hline KL & $\mathrm{M}$ & $9.7 \pm 1.24 \mathrm{~b}$ & $11.2 \pm 1,92 \mathrm{a}$ & $9.9 \pm 1.65 b$ & $9.7 \pm 1.33 b$ & $10.1 \pm 1.56$ & $9.8 \pm 1.36$ & $* * *$ & $* * *$ & ns \\
\hline & $\mathrm{F}$ & $9.3 \pm 1.11 \mathrm{~b}$ & $10.42 \pm 1.48 \mathrm{a}$ & $9.2 \pm 1.20 \mathrm{~b}$ & $9.1 \pm 1.18 \mathrm{~b}$ & $9.6 \pm 1.28$ & & & & \\
\hline $\mathrm{BC}$ & $\mathrm{M}$ & $36.5 \pm 3.70 b$ & $38.7 \pm 4.43 \mathrm{a}$ & $37.3 \pm 4.41 \mathrm{ab}$ & $35.9 \pm 4.22 b$ & $37.2 \pm 4.18$ & $36.1 \pm 3.72$ & $* * *$ & $* * *$ & ns \\
\hline & $\mathrm{F}$ & $34.9 \pm 2.84 \mathrm{c}$ & $37.1 \pm 3.77 \mathrm{a}$ & $35.9 \pm 3.74 b$ & $34.4 \pm 3.48 \mathrm{c}$ & $35.8 \pm 3.52$ & & & & \\
\hline NL & $\mathrm{M}$ & $12.8 \pm 1.64 \mathrm{a}$ & $12.6 \pm 3.78 \mathrm{a}$ & $13.1 \pm 2.12 \mathrm{a}$ & $13.2 \pm 1.68 \mathrm{a}$ & $12.9 \pm 2.50$ & $11.9 \pm 2.24$ & $* * *$ & $* * *$ & ns \\
\hline & $\mathrm{F}$ & $11.8 \pm 1.41 \mathrm{a}$ & $10.8 \pm 2.09 \mathrm{~b}$ & $11.8 \pm 3.08 \mathrm{a}$ & $12.0 \pm 1.44 \mathrm{a}$ & $11.5 \pm 2.13$ & & & & \\
\hline TL & $\mathrm{M}$ & $18.3 \pm 3.84 \mathrm{a}$ & $19.5 \pm 5.39 \mathrm{a}$ & $18.5 \pm 4.87 \mathrm{a}$ & $18.8 \pm 5.42 \mathrm{a}$ & $18.8 \pm 4.88$ & $16.3 \pm 3.10$ & Ns & $* * *$ & ns \\
\hline & $\mathrm{F}$ & $15.1 \pm 2.03 \mathrm{~b}$ & $15.8 \pm 2.39 \mathrm{a}$ & $15.3 \pm 1.81 \mathrm{~b}$ & $14.9 \pm 2.01 \mathrm{~b}$ & $15.3 \pm 2.10$ & & & & \\
\hline Toeto L & $\mathrm{M}$ & $29.4 \pm 3.15 a$ & $30.0 \pm 3.69 a$ & $28.9 \pm 3.94 \mathrm{a}$ & $29.3 \pm 3.17 \mathrm{a}$ & $29.5 \pm 3.50$ & $27.2 \pm 2.81$ & Ns & $* * *$ & ns \\
\hline & $\mathrm{F}$ & $26.1 \pm 2.64 \mathrm{a}$ & $26.2 \pm 2.47 \mathrm{a}$ & $26.4 \pm 2.68 \mathrm{a}$ & $26.6 \pm 2.20 \mathrm{a}$ & $26.3 \pm 2.50$ & & & & \\
\hline Height & $\mathrm{M}$ & $40.9 \pm 4.92 b$ & $45.6 \pm 5.56 \mathrm{a}$ & $41.64 \pm 5.67 \mathrm{~b}$ & $41.9 \pm 5.47 \mathrm{~b}$ & $42.6 \pm 3.59$ & $38.9 \pm 4.19$ & $* * *$ & $* * *$ & ns \\
\hline & $\mathrm{F}$ & $36.0 \pm 3.38 \mathrm{c}$ & $39.5 \pm 4.05 \mathrm{a}$ & $36.9 \pm 3.90 \mathrm{~b}$ & $36.6 \pm 2.98 b c$ & $37.5 \pm 3.66$ & & & & \\
\hline BW (kg) & $\mathrm{M}$ & $1.5 \pm 0.38 \mathrm{ab}$ & $1.6 \pm 0.46 \mathrm{a}$ & $1.4 \pm 0.40 \mathrm{~b}$ & $1.5 \pm 0.41 \mathrm{~b}$ & $1.5 \pm 0.62$ & $1.4 \pm 0.34$ & $*$ & $* * *$ & * \\
\hline & $\mathrm{F}$ & $1.3 \pm 0.29 \mathrm{a}$ & $1.3 \pm 0.30 \mathrm{a}$ & $1.3 \pm 0.30 \mathrm{a}$ & $1.3 \pm 0.32 \mathrm{a}$ & $1.3 \pm 0.30$ & & & & \\
\hline
\end{tabular}

abc Means in a row with different superscript letters denote significant differences between populations or sampling districts $(\mathrm{p}<0.05)$ and ${ }^{\mathrm{P}}{ }_{\mathrm{D}}$ is effect of distrcts, ${ }_{\mathrm{S}}^{\mathrm{P}}$ is effect of sex, ${ }^{\mathrm{P}}{ }_{\mathrm{DS}}$ is interaction effect of districts and sex; ,,,

\section{Variations in qualitative traits \\ Plumage colour}

The plumage and skin colours of chicken ecotypes in the study areas are delineated in Table 2 . The present study showed that there is a variation in plumage colour among ecotypes $(\mathrm{p}<0.001)$. The predominant plumage colour for Bambassi ecotype is white (39\%) followed by black $(12.7 \%)$ and gray $(11.6 \%)$. Whereas, major plumage colours for Kamashi ecotype include black (13.5\%) followed by brown (10.5\%) and golden (10\%). The Maokomo ecotype was dominated by black $(21 \%)$ followed by gray $(17 \%)$ and then brown colour $(12 \%)$. Gray $(16.5 \%)$ was the predominant plumage colour of Homosha ecotype followed by Gebsema (9.8 \%) and Kokima $(9 \%)$. Majority $(70 \%)$ of chickens in the study areas had white skin. 
Table 2 Plumage and skin colour characteristics of indigenous chicken populations of the study areas

\begin{tabular}{|c|c|c|c|c|c|c|}
\hline \multirow[b]{3}{*}{ Morphologies } & \multicolumn{4}{|c|}{ Districts/ecotypes } & \multirow[t]{2}{*}{ Total } & \multirow[b]{3}{*}{$\mathrm{X}^{2}$-test } \\
\hline & Bambassi & Kamashi & Mao-komo & Homosha & & \\
\hline & Freq (\%) & Freq $(\%)$ & Freq $(\%)$ & Freq (\%) & Freq $(\%)$ & \\
\hline Plumage colour & & & & & & $90 * * *$ \\
\hline White & $34(18)$ & $25(9.4)$ & $13(6.6)$ & $15(7.7)$ & $87(10.3)$ & \\
\hline Golden & $4(2)$ & $27(10)$ & $9(4.6)$ & $8(4.1)$ & $48(5.7)$ & \\
\hline Wheaten & $14(7.4)$ & $11(4.1)$ & $8(4.1)$ & $15(7.7)$ & $48(5.7)$ & \\
\hline Multiple & $13(6.9)$ & $25(9.4)$ & $18(9.1)$ & $10(5.2)$ & $69(8.1)$ & \\
\hline Black & $24(12.7)$ & $36(13.5)$ & $42(21)$ & $16(8.2)$ & $118(14)$ & \\
\hline Red & $17(9)$ & $18(6.7)$ & $8(4.1)$ & $14(7.2)$ & $57(6.7)$ & \\
\hline Gebsema & $21(11)$ & $22(8.2)$ & $7(3.6)$ & $19(9.8)$ & $69(8.1)$ & \\
\hline Teterma & $4(2.1)$ & $22(8.2)$ & $10(5.1)$ & $17(8.8)$ & $53(6.3)$ & \\
\hline Brown & $15(8)$ & $29(10.5)$ & $24(12)$ & $17(8.8)$ & $81(9.6)$ & \\
\hline Kokima & $10(5.3)$ & $14(5.2)$ & $14(7.1)$ & $18(9)$ & $55(6.6)$ & \\
\hline Gray & $22(11.6)$ & $21(7.9)$ & $33(17)$ & $32(16.5)$ & $108(13)$ & \\
\hline Zigrima & $10(5)$ & $15(5.6)$ & $9(4.9)$ & $14(7.2)$ & $48(5.7)$ & \\
\hline Other & $1(0.3)$ & $3(1.1)$ & $2(1)$ & $0(0)$ & $6(0.7)$ & \\
\hline Total & $189(100)$ & $267(100)$ & $197(100)$ & 194(100) & $847(100)$ & \\
\hline Skin colour & & & & & & $56 * * *$ \\
\hline Bluish black & $1(0.5)$ & $10(3.7)$ & $2(1)$ & $1(0.5)$ & $14(1.7)$ & \\
\hline White & $156(82)$ & $184(69)$ & $106(54)$ & $142(73)$ & $588(70)$ & \\
\hline Yellow & $32(17)$ & $70(26)$ & $88(45)$ & $51(26)$ & $241(28.5)$ & \\
\hline Total & $189(100)$ & $267(100)$ & 197(100) & 194(100) & $847(100)$ & \\
\hline
\end{tabular}

Freq $=$ frequency, $\mathbf{X}^{2}=$ Pearson- chi square, asterisks $(* * *)$ indicate significant difference at $1 \%$ level of probability, Gebsema $=$ wheaten strips on black background, Teterma=Black or red speckles on white background, Kokima=white or grayish strips on brown or reddish background, Zigrima= black and white spotted feather.

\section{Neck feather, head shape, comb type, and earlobe colour}

Neck feather types, comb types, head shape and earlobe colour of local chickens in the study districts are indicated in Table 3. About $9 \%$ of the total chicken populations in the study districts were naked-neck type, though differences were observed among the populations regarding their distribution. Relatively higher proportion of naked-neck chickens were found in Kamash ecotype (13.6\%) followed by Mao-komo ecotype $(11.2 \%)$ than the other two ecotypes. The naked-neck character is described as the expression of a major gene found in local chicken populations of the tropics and is considered to have desirable effects on heat tolerance. Rather rare occurrence of naked-necked chickens might be an indication of a negative selection against this character (Horst, 1989).

Various comb types were observed in the current study where differences in proportion of comb types were manifested among the ecotypes. Single comb was the most dominant $(82 \%)$ comb type in all ecotypes. Other comb types (rose, cushion and pea) appeared in small proportion. The occurrence of varieties of different comb types observed in this study might be due to interactions of different genes responsible for comb expression has contended that the heredity of comb type in chickens is attributed to two autosomal pairs of genes RR for Rose type and PP for Pea type) ( Crowford ,1990; Imsland et al., 2012).

The dominant earlobe colours of the ecotypes were red and white (34\%) and red colour (29\%). This finding is in agreement with previous studies in indigenous chicken ectypes at different parts of Ethiopia (Embet et al., 2014; Tadelle, 2003; Duguma, 2006 and Halima, 2007). Regarding the head shape, 81\% of the chickens in the study areas had plain head shape and the remaining (19\%) were crest. Figures 1, 2, 3 and 4 show pictures of some plumage characteristics of local chickens appeared in the study areas. 


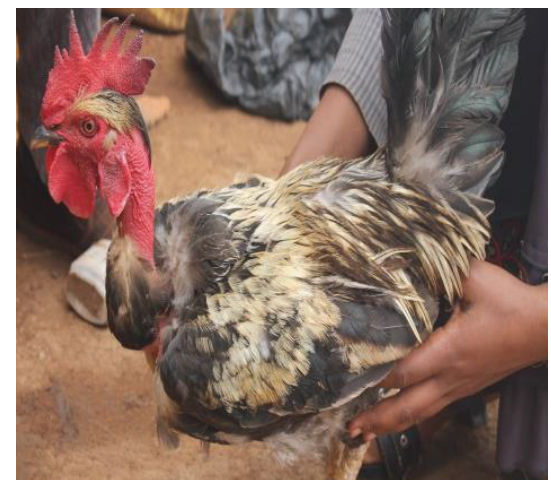

Fig 1.Naked-neck

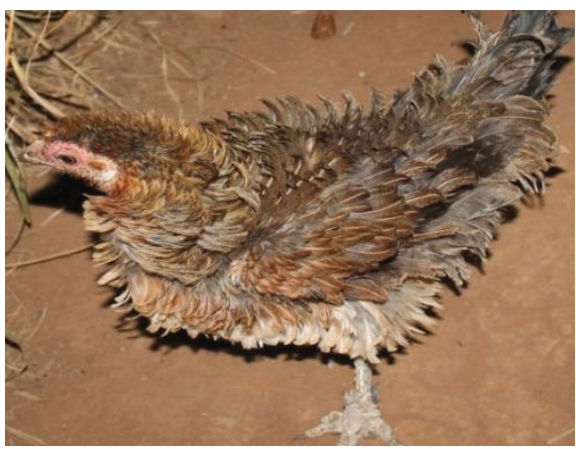

Fig.3. Frizzled feather

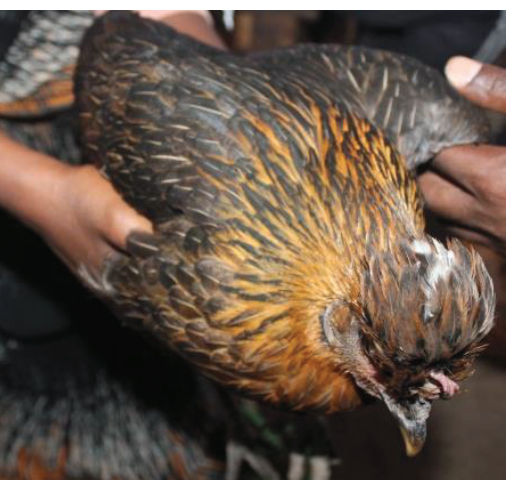

Fig 2.Crest-head

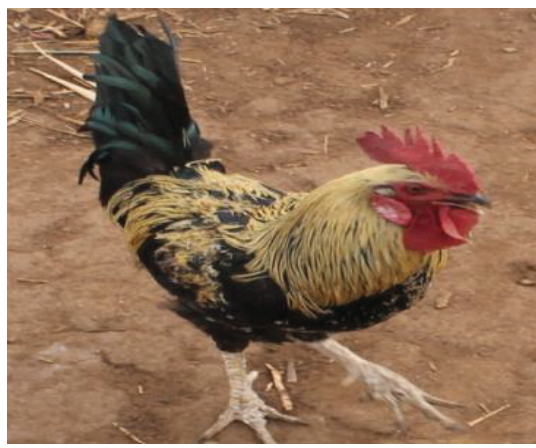

Fig 4.Normal feather

Table 3 .Morphological characteristics of the neck and head region and body shape of indigenous chicken populations in the study areas.

\begin{tabular}{|c|c|c|c|c|c|c|}
\hline \multirow{3}{*}{$\begin{array}{l}\text { Parameter } \\
\text { expression }\end{array}$} & \multicolumn{4}{|c|}{ Districts/ecotypes } & \multirow[b]{2}{*}{ Total } & \multirow[b]{2}{*}{$\mathrm{X}^{2}-$ test } \\
\hline & Bambassi & Kamashi & Mao-komo & Homosha & & \\
\hline & Freq(\%) & Freq(\%) & Freq(\%) & Freq(\%) & Freq(\%) & \\
\hline \multicolumn{2}{|l|}{ Neck feather } & & & & & $22 * * *$ \\
\hline Naked neck & 19(10) & $36(13.6)$ & $22(11)$ & $4(2.1)$ & $77(9)$ & \\
\hline Feathered neck & $170(90)$ & $231(86.4)$ & $175(89)$ & $190(98)$ & $770(91)$ & \\
\hline Total & 189(100) & $267(100)$ & 197(100) & 194(100) & $847(100)$ & \\
\hline \multicolumn{7}{|l|}{ Head shape } \\
\hline Crest & 19(10) & $54(20)$ & $38(19)$ & $47(24)$ & $158(19)$ & $13.7^{\mathrm{ns}}$ \\
\hline Plain & $170(89)$ & $213(80)$ & $159(81)$ & $147(75)$ & 687(81) & \\
\hline Total & 189(100) & $267(100)$ & 197(100) & 194(100) & $847(100)$ & \\
\hline \multicolumn{7}{|l|}{ Comp type } \\
\hline Cushion & $5(4.4)$ & $6(12)$ & $10(6)$ & $6(3)$ & $26(5)$ & $53 * * *$ \\
\hline Pea & $13(12)$ & $8(13)$ & $8(5)$ & $5(3)$ & $33(6)$ & \\
\hline Rose & $18(16)$ & $5(9)$ & $7(4)$ & $6(3)$ & $36(7)$ & \\
\hline Single & $78(64)$ & $37(66)$ & $140(85)$ & 179(91) & $434(82)$ & \\
\hline Total & $114(100)$ & $56(1000$ & $165(100)$ & $196(100)$ & $847(100)$ & \\
\hline Body shape & & & & & & $27 * * *$ \\
\hline Blocky & $84(44)$ & $182(68)$ & $123(62)$ & $114(59)$ & 503(59) & \\
\hline Triangular & $105(56)$ & $85(32)$ & $74(38)$ & $80(41)$ & $344(41)$ & \\
\hline Total & 189(100) & $267(100)$ & 197(100) & 194(100) & $847(100)$ & \\
\hline
\end{tabular}


Table 4. Variations in shank, earlobe, and eye colours among chicken ecotypes of the study areas

\begin{tabular}{|l|l|l|l|l|l|l|}
\hline \multirow{2}{*}{ Parameter expression } & \multicolumn{2}{|c|}{ Districts/ecotypes } & & \\
\cline { 2 - 6 } & Bambassi & Kamashi & Mao-komo & Homosha & Total & $X^{2}-$ test \\
\cline { 2 - 6 } & Freq (\%) & Freq (\%) & Freq (\%) & Freq (\%) & Freq (\%) & \\
\hline Shank color & & & & & $107 * * *$ \\
\hline Black & $16(8.5)$ & $44(17)$ & $48(24)$ & $14(7)$ & $122(14.4)$ & \\
\hline Bluish black & $56(30)$ & $57(21)$ & $46(23)$ & $97(50$ & $256(30)$ & \\
\hline Green & $0(0)$ & $1(0.4)$ & $4(2)$ & $1(0.5)$ & $6(.7)$ & \\
\hline Green blue & $5(3)$ & $8(3)$ & $2(1)$ & $2(1)$ & $17(2)$ & \\
\hline White & $79(42)$ & $76(29)$ & $52(26)$ & $59(31)$ & $269(32)$ & \\
\hline Yellow & $33(18)$ & $75(28)$ & $44(22)$ & $21(11)$ & $173(21)$ & \\
\hline Total & $189(100)$ & $267(100)$ & $197(100)$ & $194(100)$ & $847(100)$ & \\
\hline Earlobe colour & & & & & & $54^{* * *}$ \\
\hline Red & $62(33)$ & $49(18)$ & $52(26)$ & $78(40)$ & $241(29)$ & \\
\hline Red and white & $56(30)$ & $112(42)$ & $62(32)$ & $61(31)$ & $291(34)$ & \\
\hline White & $45(24)$ & $75(28)$ & $41(21)$ & $36(19)$ & $197(23)$ & \\
\hline Yellow & $11(6)$ & $16(6)$ & $20(10)$ & $7(4)$ & $54(6)$ & \\
\hline Black & $4(2)$ & $9(3)$ & $6(3)$ & $6(3)$ & $25(3)$ & \\
\hline Yellow and red & $11(6)$ & $6(2)$ & $14(7)$ & $6(3)$ & $37(5)$ & \\
\hline Total & $189(100)$ & $267(100)$ & $197(100)$ & $194(100)$ & $847(100)$ & \\
\hline Eye colour & & & & & & $110^{* * *}$ \\
\hline Pearl & $26(14)$ & $22(8)$ & $29(19)$ & $51(23)$ & $128(15)$ & \\
\hline Brown & $97(11)$ & $147(55)$ & $103(53)$ & $86(44)$ & $433(51)$ & \\
\hline Orange & $65(34)$ & $55(21)$ & $54(27)$ & $51(26)$ & $225(27)$ & \\
\hline Red & $0(0)$ & $11(4) 1$ & $10(5)$ & $4(2)$. & $25(3)$ & $34(4)$ \\
\hline Black & $0(0)$ & $32(12)$ & $0(0)$ & $2(1)$ & $847(100)$ & \\
\hline Total & $189(100)$ & $267(100)$ & $197(100)$ & $194(100)$ & \\
\hline
\end{tabular}

\section{Conclusion}

Indigenous chicken population in the study area had distinct physical variations for both qualitative and quantitative traits under traditional management system. This phenotypic variability caused by both genetic and environmental factors. The high phenotypic diversity in indigenous chicken is major evidence for the existence of high genetic variability in the study area. This variability may provide an opportunity for genetic improvement of chicken through selection.

\section{Reference}

Addis Getu, Kefyalew Alemayehu and Zewdu Wuletaw.2013. Phenotypic Characterization of Indigenous Chicken Ecotypes in North Gondar Zone, Ethiopia. American-Eurasian Journal of Scientific Research 8 (6): 248-255, 2013 ISSN 1818-6785.

Addisu Hailu, Zewdu Wuletaw and Hailu Mazengia.2014. Breeding practice and objective of indigenous chicken in North Wollo, Amhara regional State, Ethiopia." International Journal of livestock production 5, no. 1 (2014): 15-22.

Agide Yisma .2015. On- Farm Phenotypic Characterization of Indigenous Chicken and chicken Production Practices in North Shewa Zone, Amhara, Ethiopia Thesis Submitted to the School of Animal and Range Sciences, Haramaya University,Haramaya,Ethiopia.

AMS. 2008. Climatic data Assosa Meteorological Station (AMS), Benishangul Gumuz Regional State of Ethiopia, Assosa.

Crawford R D 1990 Poultry Breeding and Genetics. Elsevier, Amsterdam

CSA (Central Statistical Agency). 2015. Agricultural sampling survey, report on livestock and livestock characteristics for the year 2014/15, Addis Ababa, Ethiopia. Pp.37-40.

Daikwo I., Okpe A and Ocheja J. 2011. Phenotypic characterization of local chickens in Dekina. International Journal of poultry science 10(6):444-447.

Duguma R. 2010. phenotypic characterization of some indigenous chicken ecotypes of Ethiopia. Livestock Research for Rural Development. Volume 18.

Emebet Moreda, Singh, H., Sisaye, T. and Johansson, A.M., 2014. Phenotypic characterization of indigenous chicken population in South West and South part of Ethiopia. British Journal of Poultry Sciences 3 (1): 1519.

Eskindir Aklilu,, Kefelegn Kebede, Tadelle Dessie, and A. K. Banerjee. 2013. Phenotypic Characterization of 
Indigenous Chicken Population in Ethiopia International Journal of Interdisciplinary and Multidisciplinary Studies,2013, Vol 1,No.1,24-32.Available online at httt://www.ijims.com.

FAO (Food and Agricultural Organization). 2012. Phenotypic characterization of animal genetic resources. FAO Animal Production and Health Guidelines No. 11. Rome.

Feyera Bodena .2016. Phenotypic Characterization of Indigenous Chicken and Their Production System in Gobu Sayo, Bako Tibe and Danno Districts of Western Oromia, Ethiopia. Thesis Submitted to the School of Animal and Range Sciences, Haramaya University,Haramaya, Ethiopia.

Guni, F. S., \& Katule, A. M. (2013). Characterization of local chickens in selected districts of the Southern Highlands of Tanzania: I. Qualitative characters. Livestock Research for Rural Development, 25(9).

Halima, H., 2007. Phenotypic and genetic characterization of indigenous chicken populations in Northwest Ethiopia. PhD, Dissertation University of the Free State, Bloemfontein, South Africa, pp:176.

Horst, P. 1989. Native fowl as reservoir for genomes and major genes with direct and indirect effects on the adaptability and their potential for tropically orientated breeding plans. Archiv fuer Gefluegelkunde (Germany, FR).

Jansson, J. O. Edén, S., and Isaksson, O. 1985. Sexual dimorphism in the control of growth hormone secretion. Endocrine reviews, 6(2), 128-150.

Nesheim, Malden C., Richard E. Austic, and Leslie E. Card. 1979. Poultry production. No. Ed. 12. Lea \& Febiger, Philadelphin, USA.

Nigussie Dana, Tadelle Dessie, Liesbeth H. van der Waaij, and Johan AM van Arendonk. 2010. Morphological features of indigenous chicken populations of Ethiopia." Animal Genetic Resources/Resources génétiques animales/Recursos genéticos animales 46 (2010): 11-23.

Rege, J.E.O., Marshall, K., Notenbaert, A., Ojango, J.M.K. and Okeyo, A.M., 2011. Pro-poor animal improvement and breeding-What can science do?. Livestock Science, 136(1), pp.15-28.

Sölkner, J., Grausgruber, H., Okeyo, A.M., Ruckenbauer, P. and Wurzinger, M., 2008. Breeding objectives and the relative importance of traits in plant and animal breeding: a comparative review. Euphytica, 161(1-2), pp.273-282.

Tadelle D .2003. Phenotypic and genetic characterization of chicken ecotypes in Ethiopia. Ph.D. Thesis submitted to Humboldt University, Germany.

Tadelle Dessie, and Ogle B .2001. Village poultry production system in the Central Highlands of Ethiopia.Trop Anim Health Prod.33. 521-537.

Workneh, A. 1992. . Preliminary survey of indigenous goat types and husbandry practices in Southern Ethiopia. M.Sc. Thesis, Alemaya University of Agriculture,Alemaya Ethiopia. 